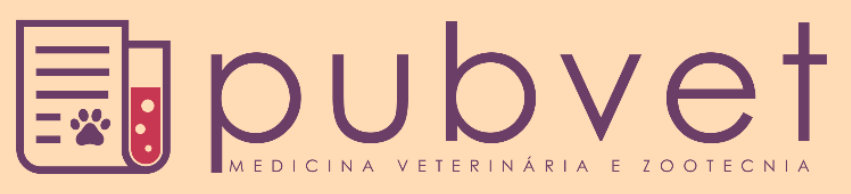

https://doi.org/10.31533/pubvet.v15n12a998.1-6

\title{
Bloqueio locorregional do plano transverso abdominal em gata submetida a ovariohisterectomia e cistotomia: Relato de caso
}

\author{
Ludimilla Cristina Teles Martins ${ }^{1 \oplus}$, Guilherme Machado Holzlsauer ${ }^{1 \oplus}$, Flávia Augusta De \\ Oliveira $^{2 \%}$, Ana Paula Gering ${ }^{3 \%}$ \\ ${ }^{I}$ Médico(a) Veterinário(a), Programa de Aprimoramento Profissional em Medicina Veterinária - Universidade Federal do \\ Tocantins, Câmpus EMVZ, Araguaína, TO, Brasil. \\ ${ }^{2}$ Médica Veterinária, Clínica Veterinária, Universidade Federal do Tocantins, Campus EMVZ, Araguaína, TO, Brasil. \\ ${ }^{3}$ Docente em Anestesiologia Veterinária, Universidade Federal do Tocantins, Campus EMVZ, Araguaína, TO, Brasil. \\ *Autor para correspondência, E-mail: ludicrismedvet@gmail.com
}

Resumo. O bloqueio do plano transverso abdominal (Tap Block) é uma técnica de anestesia locorregional que consiste na aplicação do anestésico local no plano inter-neurofascial, promovendo bloqueio da pele, músculos e peritônio parietal da parede abdominal ventral nos animais. O objetivo do trabalho foi relatar o uso do bloqueio do plano transverso abdominal em gata submetida à ovariohisterectomia e cistotomia. Foi atendida na Clínica Veterinária Universitária uma gata para atendimento pré-cirúrgico de castração. O tutor relatou que aplicava vacina anti-cio e desde então observou aumento de volume abdominal. Realizaram-se exames laboratoriais de hemograma, bioquímicos (Alanina Amino Trasnferase-ALT, fosfatase alcalina, ureia e creatinina), proteínas totais e frações e ultrassonografia abdominal, sendo que neste observou-se cálculo em vesícula urinária e piometra/hemometra/mucometra. O paciente foi encaminhado para realizar ovariohisterectomia terapêutica e cistotomia. A medicação pré-anestésica consistiu em midazolam $(0,2 \mathrm{mg} / \mathrm{kg})$, cetamina $(2 \mathrm{mg} / \mathrm{kg})$, metadona $(0,3 \mathrm{mg} / \mathrm{kg})$ e acepromazina $(0,03 \mathrm{mg} / \mathrm{kg})$ todos por via intramuscular. Vinte minutos após MPA, inseriu-se o cateter 22G na veia cefálica direita e em seguida o animal foi encaminhado para a sala de ultrassonografia para realizar o bloqueio locorregional. Posicionou-se o animal em decúbito dorsal e realizou-se a anestesia locorregional do plano transverso do abdômen guiada por ultrassom, com bupivacaína $(0,2 \mathrm{ml} / \mathrm{kg})$ diluída $1: 2 \mathrm{em}$ água para injeção distribuída em quatro pontos do abdômen. No centro cirúrgico, efetuou-se a indução anestésica com propofol dose efeito e para manutenção da anestesia, optou-se pelo sevofluorano. Durante a monitoração anestésica não se observou alterações hemodinâmicas que revelassem nocicepção no animal, devido ao uso do bloqueio locorregional. A recuperação se deu de maneira rápida, sem observar-se sinais de dor no comportamento animal, mostrando a eficácia analgésica do bloqueio e a importância do mesmo na rotina anestésica.

Palavras-chave: Bupivacaína, metadona, monitoração anestésica

\section{Locoregional blockage of the transverse abdominal plane in a female cat who underwent ovariohysterectomy and cystotomy: Case report}

\footnotetext{
Abstract. Blockade of the transverse abdominal plane (Tap Block) is a technique of locoregional anesthesia that consists of the application of local anesthetic in the interneurofascial plane, promoting blockage of the skin, muscles and parietal peritoneum of the ventral abdominal wall in animals. The aim of the study was to report the use of transverse abdominal plane block in a cat submitted to ovariohysterectomy and cystotomy.
} 
A cat was attended at the Veterinary University for pre-surgical castration care. The tutor reported that he was applying an anti-heat vaccine and has since observed an increase in abdominal volume. Laboratory blood tests, biochemical tests (Alanine Amino TrasnferaseALT, alkaline phosphatase, urea and creatinine), total proteins and fractions and abdominal ultrasound were performed, in which a calculation was observed in the urinary vesicle and pyometra/hemometra/mucometra. The patient was referred for therapeutic ovariohysterectomy and cystotomy. Pre-anesthetic medication consisted of midazolam $(0.2$ $\mathrm{mg} / \mathrm{kg})$, ketamine $(2 \mathrm{mg} / \mathrm{kg})$, methadone $(0.3 \mathrm{mg} / \mathrm{kg})$ and acepromazine $(0.03 \mathrm{mg} / \mathrm{kg})$ all intramuscularly. Twenty minutes after MPA, the $22 \mathrm{G}$ catheter was inserted into the right cephalic vein and then the animal was sent to the ultrasound room to perform the locoregional block. The animal was placed in the supine position and ultrasound-guided locoregional anesthesia of the transverse plane of the abdomen was performed with bupivacaine $(0.2 \mathrm{ml} / \mathrm{kg})$ diluted 1: 2 in water for injection distributed in four points of the abdomen. In the operating room, anesthetic induction was performed with propofol dose effect and for the maintenance of anesthesia, sevofluorane was chosen. During anesthetic monitoring, hemodynamic changes that revealed nociception in the animal were not observed, due to the use of locoregional block. Recovery took place quickly, with no signs of pain in animal behavior, showing the analgesic efficacy of the block and the importance of it in the anesthetic routine.

Keywords: Bupivacaine, methadone, anesthetic monitoring

\section{Introdução}

A anestesiologia veterinária passou por grandes mudanças nas últimas décadas, relacionadas às técnicas, fármacos e monitoração, oferecendo maior segurança na execução dos procedimentos. Ultimamente, a anestesia locorregional vem ganhando destaque por ter eficácia analgésica, promover rápida recuperação anestésica, redução da utilização de anestésicos gerais e do risco anestésico (Hemmerling, 2018). A anestesia locorregional visa extinguir a sensação de dor em uma área específica do corpo. O bloqueio dos canais de sódio, causa interrupção da geração e propagação do potencial de ação em tecidos eletricamente excitáveis, levando a diminuição de sensibilidade, mobilidade e função autonômica. A dessensibilização das fibras periféricas ocorre na seguinte sequência: primeiro as autonômicas, em seguida as responsáveis pela sensibilidade térmica, dolorosa e tátil, depois as relações de pressão e vibração e por fim as proprioceptivas e motoras (Carvalho et al., 2014; Fantoni et al., 2002; Muir et al., 1992; Paddleford, 2001).

Atualmente, o bloqueio do plano transverso abdominal (Tap Block) vem ganhando destaque na Anestesiologia Veterinária, e este é uma técnica de anestesia locorregional que consiste na aplicação do anestésico local no plano interneurofascial, promovendo bloqueio da pele, músculos e peritônio parietal da parede abdominal ventral nos animais. Este bloqueio é indicado para cesariana, laparotomia, cistotomia, histerectomia (Fernandes et al., 2021; Klaumann \& Otero, 2013; Kupiec et al., 2018).

Para evitar complicações e executar a técnica de forma efetiva, é necessário conhecimento farmacológico dos anestésicos locais, das estruturas anatômicas e do uso de ultrassonografia para localização e bloqueio seletivo de um ou mais nervos de um membro (Crevecoeur \& Barouk, 2010; Klaumann \& Otero, 2013).

O trabalho tem como objetivo relatar o caso de uma gata submetida à ovariohistectomia terapêutica e cistotomia utilizando o Tap Block para analgesia trans e pós-operatória.

\section{Relato de caso}

Foi atendido na Clínica Veterinária Universitária, no dia 4 de setembro de 2020, uma gata, sem raça definida, com um ano e seis meses, pesando $3,7 \mathrm{~kg}$. O tutor levou até a clínica com o intuito de realizar castração. Relatou que aplicava vacina anti-cio e desde a última aplicação observou aumento de volume abdominal.

Ao exame físico, observou-se mucosas normocoradas, TPC 2", frequência cardíaca de 232 batimentos por minuto, frequência respiratória de 32 movimentos por minutos, temperatura $38,6^{\circ} \mathrm{C}$, 
estado de consciência alerta e pulso forte e rítmico. Na palpação abdominal observou-se aumento em região uterina.

Foram solicitados exames complementares: hemograma, bioquímicos (ureia, creatinina, ALT, fosfatase alcalina), proteínas totais e frações e ultrassonografia abdominal, no qual observou-se presença de estrutura formadora de sombra acústica medindo 1,0 × $0,3 \mathrm{~cm}$ na vesícula urinária e útero preenchido por conteúdo anecogênico (líquido), corpo e cornos uterinos com dimensões aumentadas e paredes espessadas, revelando cálculo em vesícula urinária e piometra/hemometra/mucometra. Os demais exames complementares estavam dentro dos padrões do intervalo para a espécie.

A partir da anamnese, exame físico e complementar, o animal foi classificado como ASA II e encaminhado para procedimento cirúrgico de cistotomia e ovariohisterectomia $(\mathrm{OH})$ terapêutica. Como medicação pré-anestésica (MPA), aplicou-se midazolam $(0,2 \mathrm{mg} / \mathrm{kg})$, cetamina $(2 \mathrm{mg} / \mathrm{kg})$, metadona $(0,3 \mathrm{mg} / \mathrm{kg})$ e acepromazina $(0,03 \mathrm{mg} / \mathrm{kg})$ todos por via intramuscular. Após 20 minutos, inseriu-se cateter $22 \mathrm{G}$ na veia cefálica direita. Procedeu-se com aplicação de $0,1 \mathrm{mg} / \mathrm{kg}$ de meloxican por via intravenosa e $2,5 \mathrm{mg} / \mathrm{kg}$ de enrofloxacina por via intramuscular.

$\mathrm{O}$ animal foi encaminhado para a sala de ultrassonografia, onde realizou-se o bloqueio locorregional Tap Block após administração de propofol (dose efeito) por via endovenosa. Utilizou-se bupivacaína diluída 1:2 em água para injeção na dose de $0,2 \mathrm{ml} / \mathrm{kg}$ em quatro pontos, após posicionar o animal em decúbito dorsal, realizar tricotomia e antissepsia da região. Os bloqueios foram realizados com agulha espinhal $22 \mathrm{G}$ na região cranial a crista ilíaca, caudal à $13^{\mathrm{a}}$ costela em ambos os lados, guiado por transdutor linear posicionado perpendicularmente ao eixo longitudinal do corpo do animal.

A indução anestésica deu-se com propofol dose efeito pela via intravenosa e a manutenção com sevofluorano. Foram monitoradas a frequência cardíaca (FC), frequência respiratória (FR), pressão arterial sistólica (doppler), temperatura $\left(\mathrm{T}^{\circ}\right)$, saturação de oxihemoglobina $\left(\mathrm{SPO}_{2}\right)$ e fração expirada de $\mathrm{CO}_{2}\left(\mathrm{ETCO}_{2}\right)$, exposto no gráfico 1 .

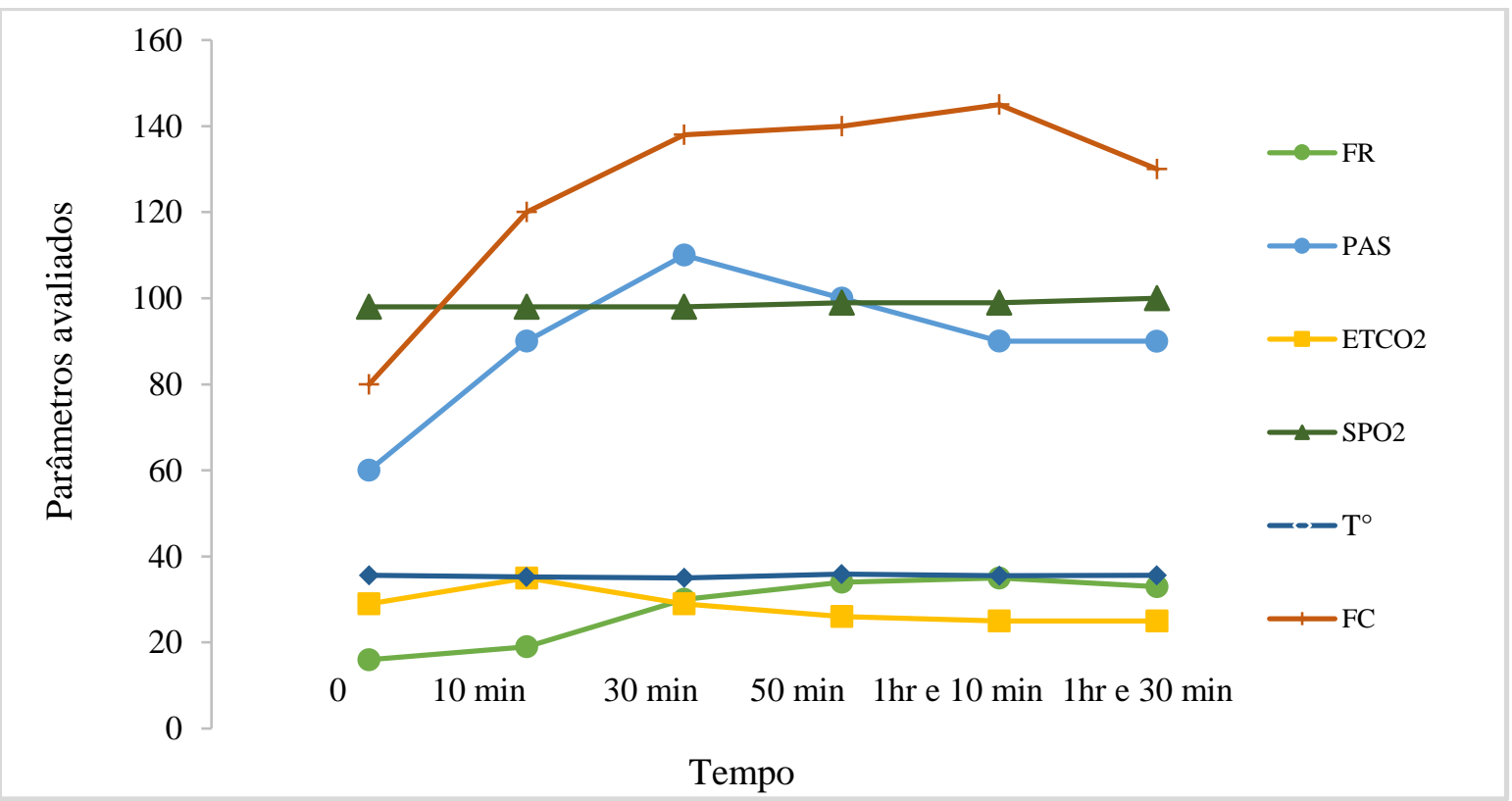

Gráfico 1. Monitoração anestésica com FC, FR, PAS, $\mathrm{ETCO}_{2}, \mathrm{SPO}_{2}$ e $\mathrm{T}^{\circ}$ de gata anestesiada com sevofluorano. Fonte: Setor de Anestesiologia Veterinária, Clínica Veterinária Universitária, 2020.

Os momentos de monitoração foram avaliados a partir da indução anestésica e marcados a cada 10 minutos. O procedimento cirúrgico durou 1 hora e 20 minutos e a anestesia 1 hora e 30 minutos. A recuperação anestésica deu-se após 30 minutos (Tabela 1). Para que ocorresse a liberação do animal a pontuação final era de 10 pontos.

Prescreveu-se como medicação pós-operatória, meloxicam $(0,1 \mathrm{mg} / \mathrm{kg})$, uma vez ao dia, por cinco dias, dipirona gotas $(25 \mathrm{mg} / \mathrm{kg})$, duas vezes ao dia, por três dias, enrofloxacina $(2,5 \mathrm{mg} / \mathrm{kg})$, duas vezes ao dia, por 10 dias todos por via oral e curativos com Furanil ${ }^{\circledR}$ spray. 
Tabela 1. Avaliação dos parâmetros de recuperação anestésica de gata submetida à cistotomia e ovariohisterectomia terapêutica

\begin{tabular}{lccccc}
\hline \multirow{2}{*}{ Parâmetros } & \multirow{2}{*}{$\begin{array}{c}\text { Chegada do animal a sala } \\
\text { de recuperação anestésica }\end{array}$} & $\begin{array}{c}30 \text { minutos após início } \\
\text { da monitoração }\end{array}$ & \multicolumn{3}{c}{ Parâmetros de referência, pontos } \\
\cline { 4 - 6 } & 0 & 1 & Alerta & Responsivo & Não responsivo \\
Consciência & 1 & 1 & - & Ausência & Presença \\
Excitação & 2 & 2 & Forte & Fraco & Filiforme \\
Pulso & 2 & 2 & Rósea & Pálida & Cianótica \\
Mucosa & 2 & 2 & $>10 \mathrm{mpm}$ & $<10 \mathrm{mpm}$ & Dispneia/Taquipneia \\
FR & 0 & 1 & $>37,5^{\circ} \mathrm{C}$ & $36,5^{\circ} \mathrm{C}-37,5^{\circ} \mathrm{C}$ & $<36,5^{\circ} \mathrm{C}$ \\
Temperatura & 8 & 10 & - & - & - \\
\hline Total & & & - & -
\end{tabular}

Fonte: Setor de Anestesiologia Veterinária, Clínica Veterinária Universitária, 2020.

\section{Resultados e discussão}

A piometra ou complexo hiperplasia endometrial cística é uma enfermidade grave do útero que acomete principalmente cadelas, ocorrendo em qualquer fase do ciclo estral. Na espécie felina, a ocorrência está relacionada principalmente à administração exógena de estrógeno e progesterona, fato que condiz com o relato de caso. Os sinais clínicos da urolitíase variam conforme a quantidade, tamanho e localização do urólito. Os mais comuns são: polaciúria, disúria e hematúria, porém em alguns casos os animais são assintomáticos. No relato de caso, a proprietária não informou quaisquer alterações no sistema urinário do animal (Carvalho et al., 2014; Fossum, 2014; Muniz Neta \& Munhoz, 2008; Rick et al., 2017).

As formas de diagnóstico para ambas as enfermidades citadas são: histórico clínico, diagnóstico por imagem (radiografia e ultrassonografia), hemograma e bioquímicos séricos. Destes, realizou-se como exames complementares, hemograma, bioquímicos séricos e a ultrassonografia. Uma vez que eram de fácil acesso na rotina clínica do estabelecimento e foram autorizados pelo responsável do animal (Jericó et al., 2015).

No caso descrito, o paciente realizou cistotomia e $\mathrm{OH}$ terapêutica utilizando o bloqueio locorregional Tap Block. Desde 2007, o Tap Block guiado por ultrassom vem sendo testado em humanos, revelando um conflito de resultados com relação a eficácia analgésica. A técnica foi então expandida para a Medicina Veterinária e comparada com outros bloqueios loco regionais (Baeriswyl et al., 2015).

De acordo com Teixeira et al. (2018) o animal é colocado em decúbito lateral e há a deposição do anestésico local no plano neurofascial, permitindo bloqueio efetivo dos nervos que inervam a parede abdominal anterior que existe no espaço entre o músculo transverso abdominal e o oblíquo abdominal interno. A parede do abdômen é dividida em três camadas musculares: oblíquo abdominal externo, oblíquo abdominal interno e transverso do abdômen, além de suas bainhas fasciais associadas. No caso descrito o animal foi posicionado em decúbito dorsal, não prejudicando a aplicação do anestésico nem a execução da técnica, localizando-se todos os grupos musculares citados (Campoy et al., 2010).

Os parâmetros vitais foram avaliados logo após a indução anestésica, e observou-se diminuição na frequência cardíaca, pressão arterial e frequência respiratória sendo justificada pelo uso do propofol na indução e da metadona na MPA. O primeiro fármaco potencializa a ação do GABA em receptores $\mathrm{GABA}_{\mathrm{A}}$, levando à hipotensão e depressão respiratória em curto período de tempo. $\mathrm{O}$ segundo é um agonista que inibe a recaptação de monoaminas e tem ação antagonista em receptores N-metil-Daspartato (NMDA), sendo utilizado em dores agudas e crônicas, podendo causar bradicardia (Fantoni et al., 2002; Fantoni \& Mastrocinque, 2005; Romeu et al., 2019; Scarparo et al., 2020).

A pressão arterial do animal se manteve entre $90 \mathrm{mmHg}$ e $110 \mathrm{mmHG}$. A estabilidade da pressão arterial sistêmica é justificada pelo uso do Tap Block, uma vez que Jakobsson et al. (2015) demonstraram que este bloqueio reduziu não apenas o uso de opioides nas primeiras 24 a 48 horas após o procedimento cirúrgico, mas também as necessidades de anestésicos no trans-operatório.

Outras técnicas de bloqueios loco regionais poderiam ser aplicadas neste tipo de cirurgia como a anestesia epidural, por exemplo. Essa técnica foi utilizada em conjunto com o Tap Block por Baeriswyl et al. (2015) e o mesmo concluiu que a adição do Tap Block não forneceu efeito analgésico adicional. $\mathrm{O}$ 
bloqueio do plano transverso abdominal de forma isolada reduziu o consumo de opioides por seis horas em cirurgias de laparotomia e laparoscopia abdominal e cesariana. Optou-se pela realização do Tap Block buscando analgesia pós-operatória, diminuição do uso de opioides e anestésicos transoperatório e interesse em praticar a técnica.

De acordo com Polydoro et al. (2006), o $\mathrm{ETCO}_{2}$ é registrado pela capnografia; os valores comuns para cães e gatos é de $35-45 \mathrm{mmHg}$. A hipocapnia indica nível leve de anestesia, hipoxemia, hipertermia, hipotensão, entre outros. No caso relatado o $\mathrm{ETCO}_{2}$ do animal se manteve entre $25-35 \mathrm{mmHg}$, sugerindo em conjunto com a frequência respiratória elevada (32-35), que o animal estava em plano anestésico superficial, fato justificado pela analgesia multimodal advinda do bloqueio locorregional durante o procedimento cirúrgico. Os demais parâmetros avaliados na monitoração anestésica mantiveram-se dentro do padrão para a espécie. A hipotermia, notada desde o início da anestesia pode ser justificada pelo uso da acepromazina na MPA. A mesma leva ao bloqueio dos receptores dopaminérgicos do sistema tuberoinfundibular, levando à hipotermia (Spinosa et al., 2006).

De acordo com Bornkamp et al. (2016) a hipotermia pós-operatória atinge 97,5\% de gatos e 83,6\% de cães e as complicações incluem diminuição do metabolismo de fármacos, aumento da dor incisional por tremores, arritmias cardíacas, aumento de sangramento, infecções de feridas pós-operatórias e aumento do tempo da recuperação anestésica. Apesar do animal ter predisposição à hipotermia, as alterações citadas acima não foram observadas.

Skouropoulou et al. (2018) desenvolveram um experimento com 20 gatas submetidas a ovariectomia, 10 receberam o Tap Block e 10 eram grupo controle. Após o procedimento cirúrgico, foi avaliado escore de dor nos animais, todos do grupo controle receberam metadona durante as primeiras $24 \mathrm{hs}$ e o grupo do Tap Block não receberam. Os autores concluíram que a técnica fornece analgesia por até 24 horas após o procedimento cirúrgico em gatas submetidas a ovariectomia.

No relato, o animal chegou na sala de recuperação anestésica não responsivo a estímulos, ausência de excitação e secreção, pulso forte, mucosa rósea, FR maior que $10 \mathrm{mpm}$ e temperatura de $35,6^{\circ} \mathrm{C}$. Para receber alta, a pontuação final deveria ser 10 e a pontuação inicial foi de 8 pontos conforme tabela 1. Após 30 minutos da avaliação o animal já havia atingido os 10 pontos. Além disso, não foi necessário o resgate analgésico durante a monitoração.

A partir do estudo citado e da descrição da recuperação anestésica, pode-se notar que a utilização do bloqueio plano transverso abdominal é importante para analgesia multimodal, não necessitando de resgate analgésico no transoperatório e pós-operatório imediato, a recuperação anestésica é rápida e é um bloqueio essencial em cirurgias abdominais.

\section{Conclusão}

O bloqueio locorregional foi eficaz para antinocepção nos períodos citados, pois não se observou alterações nos parâmetros do animal durante a cirurgia e o mesmo teve recuperação anestésica rápida, sem a necessidade de resgate analgésico e sinais de dor, como incômodo na incisão cirúrgica.

\section{Referências bibliográficas}

Baeriswyl, M., Kirkham, K. R., Kern, C., \& Albrecht, E. (2015). The analgesic efficacy of ultrasoundguided transversus abdominis plane block in adult patients: a meta-analysis. Anesthesia \& Analgesia, 121(6), 1640-1654.

Bornkamp, J. L., Robertson, S., Isaza, N. M., Harrison, K., DiGangi, B. A., \& Pablo, L. (2016). Effects of anesthetic induction with a benzodiazepine plus ketamine hydrochloride or propofol on hypothermia in dogs undergoing ovariohysterectomy. American Journal of Veterinary Research, 77(4), 351-357.

Campoy, L., Bezuidenhout, A. J., Gleed, R. D., Martin-Flores, M., Raw, R. M., Santare, C. L., Jay, A. R., \& Wang, A. L. (2010). Ultrasound-guided approach for axillary brachial plexus, femoral nerve, and sciatic nerve blocks in dogs. Veterinary Anaesthesia and Analgesia, 37(2), 144-153. 
Carvalho, B., Fritzen, E. L., Parodes, A. G., Santos, R. B., \& Gedoz, L. (2014). O emprego dos anestésicos locais em odontologia: Revisão de literatura. Revista Brasileira de Odontologia, 70(2), 178.

Crevecoeur, A., \& Barouk, D. (2010). Anestesia locorregional. EMC-Tratado de Medicina, 14(2), 1-8.

Fantoni, D. T., \& Mastrocinque, S. (2005). Analgesia preventiva. In P. E. Otero (Ed.), Dor: Avaliação e tratamento em pequenos animais (pp. 76-80). Interbook.

Fantoni, D. T., Mastrocinque, S., Fantoni, D. T., \& Cottopassi, S. R. (2002). Fisiopatologia e controle da dor. In D. T. Fabntoni (Ed.), Anestesia em cães e gatos (pp. 323-336). Roca.

Fernandes, H. S., Azevedo, A. S., Ferreira, T. C., Santos, S. A., Rocha Filho, J. A., \& Vieira, J. E. (2021). Ultrasound-guided peripheral abdominal wall blocks. Clinics, 76.

Fossum, T. W. (2014). Cirurgia de pequenos animais (4th ed., Vol. 1). Elsevier Brasil.

Hemmerling, T. M. (2018). Pain management in abdominal surgery. Langenbeck's Archives of Surgery, 403(7), 791-803.

Jakobsson, J., Wickerts, L., Forsberg, S., \& Ledin, G. (2015). Transversus abdominal plane (TAP) block for postoperative pain management: a review. F1000Research, 4.

Jericó, M. M., Kogika, M. M., \& Andrade Neto, J. P. (2015). Tratado de medicina interna de cães e gatos. Guanabara Koogan.

Klaumann, P. R., \& Otero, P. E. (2013). Anestesia locorregional em pequenos animais. Roca.

Kupiec, A., Zwierzchowski, J., Kowal-Janicka, J., Goździk, W., Fuchs, T., Pomorski, M., Zimmer, M., \& Kübler, A. (2018). The analgesic efficiency of transversus abdominis plane (TAP) block after caesarean delivery. Ginekologia Polska, 89(8), 421-424.

Muir, W. W., Hubbell, J. A. E., Skarda, R. T., \& Bednarski, M. R. (1992). Manual de anestesia veterinária. Acribia.

Muniz Neta, E. dSouza, \& Munhoz, A. D. (2008). Urolitíase em cães e gatos: uma revisão. MEDVEP. Revista Científica de Medicina Veterinária, 6(17), 24-34.

Paddleford, R. R. (2001). Manual de anestesia em pequenos animais. Roca.

Polydoro, A. S., Natalini, C. C., Raiser, A. G., \& Hennemanin, C. R. A. (2006). Analgesia e anestesia epidural em cães e gatos: revisão de literatura. A Hora Veterinária, 25(149), 31-37.

Rick, G. W., Conrad, M. L. H., Vargas, R. M., Machado, R. Z., Lang, P. C., Serafini, G. M. C., \& Bones, V. C. (2017). Urolitíase em cães e gatos. PUBVET, 11, 646-743.

Romeu, R., Gorczak, R., \& Valandro, M. A. (2019). Analgesia farmacológica em pequenos animais. PUBVET, 13, 1-11.

Scarparo, V. A., Gorczak, R., \& Valandro, M. A. (2020). Anesthesia in high-risk patients: an anesthetic approach to cardiac, nephropathic, hepatic, pediatric and senile patients. Veterinaria Em Foco, 17(2), $12-26$.

Skouropoulou, D., Lacitignola, L., Centonze, P., Simone, A., Crovace, A. M., \& Staffieri, F. (2018). Perioperative analgesic effects of an ultrasound-guided transversus abdominis plane block with a mixture of bupivacaine and lidocaine in cats undergoing ovariectomy. Veterinary Anaesthesia and Analgesia, 45(3), 374-383.

Spinosa, H. S. de S., Górniak, S. L., \& Bernardi, M. M. (2006). Farmacologia aplicada à medicina veterinária. Koogan Guanabara.

Teixeira, L. G., Pujol, D. M., Pazzim, A. F., Souza, R. P., \& Fadel, L. (2018). Combination of Transversus abdominis plane block and Serratus plane block anesthesia in dogs submitted to masctetomy. Pesquisa Veterinária Brasileira, 38, 315-319.

Histórico do artigo:

Recebido: 14 de agosto de 2021

Aprovado: 17 de setembro de 2021

Disponível on-line: 22 de novembrode 2021
Licenciamento: Este artigo é publicado na modalidade Acesso Aberto sob a licença Creative Commons Atribuição 4.0 (CC-BY 4.0), a qual permite uso irrestrito, distribuição, reprodução em qualquer meio, desde que o autor e a fonte sejam devidamente creditados. 